\title{
Heterosis Studies for Yield and Yield Attributing Characters of Tomato (Solanum lycopersicum L.)
}

\author{
Jyothi Kattegoudar $^{1^{*}}$, H.B. Lingaiah ${ }^{2}$, N.C. Mamtha ${ }^{3}$, Ambresh $^{4}$ and B. Arunkumar ${ }^{4}$ \\ ${ }^{1}$ Department of Vegetable Science, College of Horticulture, University of Horticultural \\ Sciences Campus, G.K.V.K. (Post), Bengaluru-65, Karnataka, India \\ ${ }^{2}$ Director of Education, University of Horticultural Sciences, Bagalkot, Karnataka, India \\ ${ }^{3}$ Department of Vegetable Science, Hissar Agricultural University, Hissar, Haryana, India \\ ${ }^{4}$ Department of Vegetable Science, University of Horticultural Sciences, \\ Bagalkot, Karnataka, India \\ *Corresponding author
}

\section{A B S T R A C T}

\section{Keywords}

Heterosis, Tomato,

Yield and yield

attributing

characters

\section{Article Info}

Accepted:

10 December 2017

Available Online:

10 January 2018

\begin{abstract}
A $8 \times 8$ full diallel cross of tomato was evaluated with parents and cheks for heterotic manifestation of yield and yield attributing characters. The heterosis over commercial check-2 to the extent of $107.08,59.79,-13.59,174.70$ and 13.58 per cent was recorded for plant height at $60 \mathrm{DAP}$, number of branches per plant 60 DAT, days to fifty per cent flowering, number of fruits per plant and average fruit weight respectively. The crosses showing heterosis for yield per plant were not heterotic for all the characters under study. The heterosis for yield was generally accompanied by heterosis for yield components. Promising crosses for yield per plant are Vaibhav x PKM-1, PKM-1 x Anagha and Arka Sourabh x Anagha, for average fruit weight (more than $70 \mathrm{~g}$ ), Vaibhav x Arka Saurabh and Vaibhav x PKM-1 crosses found promising with high standard heterosis. The yield and yield components indicated the role of both additive and non-additive gene effect in governing these traits.
\end{abstract}

\section{Introduction}

Tomato (Solanum lycopersicum L.) is one of the most important solanaceous vegetable crops grown widely all over the world. It is a very versatile vegetable for culinary purposes ranging from ripe fresh fruit to range of processed products. It is native of Peru Ecuador region. Now, cultivation of tomato has become increasingly popular since midnineteenth century. So far efforts of many vegetable breeders from both public and private sector have resulted in spectacular improvement in yield characters. As a result of these efforts, hundreds of new cultivars have been developed since 50 years to meet the diverse needs and varied situations and climates under which tomato grows. Exploitation of heterosis is a quick and convenient way of combining desirable traits from contrasting parents. Keeping aforesaid information in view, a study on tomato was 
initiated at College of Horticulture, GKVK, Bengaluru with the objective to estimate the heterosis of crosses for yield and yield attributing characters in tomato.

\section{Materials and Methods}

The study on "Heterosis for yield and yield attributing characters in tomato (Solanum lycopersicum L.)" was undertaken during the year 2011-2013. Full diallel analysis involving four bacterial wilt resistance genotypes namely Arka Abha, Utkal Pragnya, Anagha and Utkal Raja and four bacterial wilt susceptible genotypes having good horticultural properties viz., Arka Meghali, Arka Saurabha, PKM-1 and Vaibhav were used in the present study. Hand emasculation and pollination technique was followed to develop $56 \mathrm{~F}_{1} \mathrm{~s}$.

\section{Results and Discussion}

Heterosis is manifested through greater vigour of $F_{1}$ over their parents resulting into higher yield through their component characters. Heterosis breeding provides opportunity for improvement in productivity, earliness, uniformity, quality and development for resistance to diseases and pest. The recent trend in tomato breeding has been towards development of hybrids to meet the specific uses (viz., earliness, growth habit, disease or pest resistance, fruit quality for processing fresh market etc.) coupled with high yield as it may be difficult to develop a hybrid having all the characters.

For plant height at 60 days after transplanting, thirty three crosses exhibited significant positive economic heterosis over check-2. Twenty-four crosses exhibited standard heterosis over the check-1. Seventeen crosses showed significant positive hetrobeltosis. Mid-parent heterosis was observed in majority of crosses. In the earlier studies also, heterosis was reported for plant height by Fageria et al., (2001) and Thakur et al., (2004). At 60 DAT, Utkal Raja x PKM-1 showed maximum positive significant heterois for number of branches per plant.

Days to 50 per cent flowering are one of the important components for earliness. Seven crosses out of fifty six showed negative economic heterosis over check-2 and over check-1.

Twelve crosses exhibited significantly negative heterosis indicating early flowering in hybrids. While fifteen crosses showed negative hetrosis over better and mid-parents. Similar results were observed by Kulkarni, (2003), Mahendrakar, (2004), and Duhan et al., (2005).

Out of 56 hybrids, 42 showed positive heterosis over check-2 and 23 over check-1 exhibited positive heterosis. Three hybrids showed positive significant heterosis over mid-parent.

The magnitude of heterosis over check-1 and check-2 for fruits per plant was as high as 123.90 per cent (PKM-1 x Anagha) and 193.76 per cent (PKM-1 x Anagha). For this character negative and positive heterosis was observed by Thakur et al., (2004).

The hybrids in general had lower average fruit weight compared to commercial checks. Hybrid between Vaibhav x Arka Sourabh with $79.16 \mathrm{~g}$ is regarded as best. The numbers of hybrids exhibited significant positive heterosis over check-1 were three and two over check-2 while those showing the negative heterosis were higher.

It gives clear indication that the character is governed by additive gene effects. Similar results were reported by Thakur et al., (2004) and Tiwari and Lal (2004) (Table 1 and 2). 
Table.1 Estimation of mid parent, better parent and standard heterosis of $8 x 8$ full diallel crosses in tomato for plant height, number of branches and days to 50 per cent flowering

\begin{tabular}{|c|c|c|c|c|c|c|c|c|c|c|c|c|c|}
\hline \multirow{2}{*}{$\begin{array}{c}\text { Sl.No } \\
.\end{array}$} & \multirow[t]{2}{*}{ Hybrids } & \multicolumn{4}{|c|}{ Plant height $(\mathrm{cm})$ at 60 DAP } & \multicolumn{4}{|c|}{ Number of branches at 60 DAP } & \multicolumn{4}{|c|}{50 per cent flowering } \\
\hline & & MP & BP & Check1 & Check2 & MP & BP & Check1 & Check2 & MP & BP & Check1 & Check2 \\
\hline 1 & Vaibhav x PKM-1 & $45.13 * *$ & $34.43 *$ & $49.09 * *$ & $70.83 * *$ & -7.82 & $-25.97 * *$ & -7.29 & -3.87 & -2.16 & -2.25 & 0.46 & 3.33 \\
\hline 2 & Vaibhav x Arka Meghali & $31.62 *$ & 26.23 & $40.00 * *$ & $60.42 * *$ & -3.62 & $-22.58 *$ & -3.04 & 0.54 & $-8.17 *$ & $-9.91 * *$ & $-7.58 *$ & -4.94 \\
\hline 3 & Vaibhav x Anagha & 5.07 & -6.56 & 3.63 & 18.75 & 0.86 & -11.59 & 10.73 & 14.81 & $-10.86^{* *}$ & $-11.26 * *$ & $-8.96 *$ & -6.37 \\
\hline 4 & Vaibhav x Utkal Pragnya & 1.08 & -0.57 & 10.27 & 26.35 & -16.77 & $-32.02 * *$ & -14.86 & -11.72 & 1.92 & 0.54 & 3.14 & 6.08 \\
\hline 5 & Vaibhav x Arka Abha & $40.83 * *$ & $39.67 * *$ & $54.91 * *$ & $77.50 * *$ & -8.09 & $-31.01 * *$ & -13.60 & -10.41 & -0.27 & -0.63 & 1.94 & 4.85 \\
\hline 6 & Vaibhav x Arka Saurabh & -16.33 & -18.60 & -4.55 & 9.38 & $-25.36 * *$ & $-39.99 * *$ & $-24.84 *$ & -22.07 & 4.85 & 1.35 & 3.97 & 6.94 \\
\hline 7 & Vaibhav x Utkal Raja & 0.75 & -0.90 & 9.91 & 25.94 & $-30.20 * *$ & $-35.00 * *$ & -18.59 & -15.59 & -0.73 & -1.89 & 0.65 & 3.52 \\
\hline 8 & PKM-1 x Arka Meghali & $36.11 * *$ & $31.25^{*}$ & $33.64 *$ & $53.13^{* *}$ & $-20.97^{*}$ & -21.00 & $-26.39 *$ & $-23.68 *$ & $-13.03 * *$ & $-14.75^{* *}$ & $-12.38 * *$ & $-9.89 * *$ \\
\hline 9 & PKM-1 x Anagha & $42.71 * *$ & $36.54^{*}$ & 29.09 & $47.92 * *$ & -8.44 & -16.00 & -6.31 & -2.86 & $-16.82 * *$ & $-17.27 * *$ & $-14.97 * *$ & $-12.55 * *$ \\
\hline 10 & PKM-1 x Utkal Pragnya & $44.14 * *$ & $35.59 *$ & $45.45 * *$ & $66.67 * *$ & 5.44 & 3.57 & 0.00 & 3.69 & $-17.52 * *$ & $-18.71^{* *}$ & $-16.45 * *$ & $-14.07 * *$ \\
\hline 11 & PKM-1 x Arka Abha & $39.29 * *$ & $30.00 *$ & $41.82 * *$ & $62.50 * *$ & 11.57 & 3.70 & -3.44 & 0.12 & 2.26 & 1.80 & 4.62 & 7.60 \\
\hline 12 & PKM-1 x Arka Saurabh & 17.81 & 6.40 & 24.77 & $42.97 *$ & 7.32 & 7.19 & 0.06 & 3.75 & 4.28 & 0.72 & 3.51 & 6.46 \\
\hline 13 & PKM-1 x Utkal Raja & $71.89 * *$ & $68.51 * *$ & $73.64 * *$ & $101.88^{* *}$ & -13.56 & $-24.64 * *$ & -5.62 & -2.14 & -6.01 & -7.19 & -4.62 & -1.90 \\
\hline 14 & Arka Meghali x Anagha & 23.67 & 14.29 & 16.36 & 33.33 & -9.70 & -17.13 & -7.57 & -4.16 & -3.69 & -5.09 & -3.51 & -0.76 \\
\hline 15 & Arka Meghali x Utkal Pragnya & 22.77 & 19.64 & 28.35 & $47.06^{* *}$ & -0.21 & -1.96 & -5.34 & -1.84 & 2.79 & 2.22 & 2.03 & 4.94 \\
\hline 16 & Arka Meghali x Arka Abha & 10.11 & 6.44 & 16.12 & 33.05 & -3.05 & -9.91 & -16.06 & -12.97 & $-16.22 * *$ & $-17.51 * *$ & $-15.99 * *$ & $-13.59 * *$ \\
\hline 17 & Arka Meghali x Arka Saurabh & 21.51 & 13.50 & $33.11^{*}$ & $52.52 * *$ & 2.92 & 2.83 & -4.02 & -0.48 & 2.66 & 1.12 & -0.18 & 2.66 \\
\hline 18 & Arka Meghali x Utkal Raja & 21.30 & 18.22 & 26.82 & $45.31 * *$ & -10.69 & $-22.13 *$ & -2.47 & 1.13 & $8.18^{*}$ & 7.38 & $7.58 *$ & $10.65^{* *}$ \\
\hline 19 & Anagha x Utkal Pragnya & 9.11 & -1.53 & 5.64 & 21.04 & -9.73 & -15.79 & -6.08 & -2.62 & 1.10 & 0.18 & 1.85 & 4.75 \\
\hline 20 & Anagha x Arka Abha & $28.93^{*}$ & 15.50 & 26.00 & $44.38 *$ & 0.06 & -14.09 & -4.19 & -0.65 & $-9.17 * *$ & $-9.26^{*}$ & $-7.58 *$ & -4.94 \\
\hline 21 & Anagha x Arka Saurabh & 15.63 & 0.39 & 17.73 & $34.90 *$ & -14.48 & $-21.45^{*}$ & -12.39 & -9.16 & -6.37 & $-9.09 *$ & $-7.58 *$ & -4.94 \\
\hline 22 & Anagha x Utkal Raja & $84.69 * *$ & $64.75^{* *}$ & $78.18 * *$ & $107.08 * *$ & $-19.41 *$ & $-23.82 * *$ & -4.59 & -1.07 & $-7.51^{*}$ & $-8.18^{*}$ & -6.65 & -3.99 \\
\hline 23 & Utkal Pragnya x Arka Abha & 22.18 & 21.17 & $32.18^{*}$ & $51.46^{* *}$ & 4.19 & -4.75 & -8.03 & -4.64 & -3.48 & -4.45 & -2.68 & 0.10 \\
\hline 24 & Utkal Pragnya x Arka Saurabh & $-32.79 * *$ & $-35.66 * *$ & -24.55 & -13.54 & $-25.68 *$ & $-26.92 *$ & $-29.43 * *$ & $-26.83^{*}$ & -4.73 & -6.67 & -6.84 & -4.18 \\
\hline 25 & Utkal Pragnya x Utkal Raja & $33.47 * *$ & $33.47^{*}$ & $43.18 * *$ & $64.06^{* *}$ & -5.85 & -16.63 & 4.42 & 8.27 & 2.31 & 2.12 & 2.31 & 5.23 \\
\hline 26 & Arka Abha x Arka Saurabh & $38.88 * *$ & $34.03 * *$ & $57.18^{* *}$ & $80.10^{* *}$ & 10.36 & 2.46 & -4.36 & -0.83 & -4.68 & $-7.53^{*}$ & -5.82 & -3.14 \\
\hline 27 & Arka Abha x Utkal Raja & $53.65^{* *}$ & $52.37 * *$ & $66.22 * *$ & $90.46^{* *}$ & $35.87 * *$ & 11.31 & $39.41 * *$ & $44.56 * *$ & -4.85 & -5.63 & -3.88 & -1.14 \\
\hline 28 & Arka Saurabh x Utkal Raja & $27.81^{*}$ & 22.36 & $43.50 * *$ & $64.43 * *$ & -15.49 & $-26.25 * *$ & -7.63 & -4.22 & 4.91 & 2.58 & 2.77 & 5.70 \\
\hline 29 & PKM-1 x Vaibhav & -5.31 & -12.30 & -2.73 & 11.46 & $-20.59 *$ & $-26.08^{*}$ & -20.14 & -17.19 & $-12.03 * *$ & $-12.10 * *$ & $-9.67 * *$ & -7.09 \\
\hline 30 & Arka Meghali x Vaibhav & 30.77 & 25.41 & 39.09 & $59.38 * *$ & -7.21 & -13.60 & -6.66 & -3.21 & $-10.84 * *$ & $-12.52 * *$ & $-10.26^{* *}$ & $-7.70^{*}$ \\
\hline
\end{tabular}


Int.J.Curr.Microbiol.App.Sci (2018) 7(1): 1073-1080

\begin{tabular}{|c|c|c|c|c|c|c|c|c|c|c|c|c|c|}
\hline 31 & Arka Meghali x PKM-1 & 11.76 & 7.77 & 9.73 & 25.73 & 8.41 & 8.37 & 0.98 & 4.7 & -3.85 & -5.76 & -3.14 & -0.38 \\
\hline 32 & Anagha x Vaibhav & 24.61 & 10.82 & 22.91 & $40.83^{*}$ & -18.68 & -19.96 & -10.73 & -7.44 & -4.43 & -4.86 & -2.40 & 0.38 \\
\hline 33 & Anagha x PKM-1 & $54.02 * *$ & $47.36 * *$ & $39.32 *$ & $59.64 * *$ & -5.52 & -13.32 & -3.33 & 0.24 & $-12.48 * *$ & $-12.95 * *$ & $-10.54 * *$ & $-7.98 *$ \\
\hline 34 & Anagha x Arka Meghali & 6.67 & -1.43 & 0.36 & 15.00 & -10.48 & -17.85 & -8.38 & -5.00 & -0.37 & -1.82 & -0.18 & 2.66 \\
\hline 35 & Utkal Pragnya x Vaibhav & 22.50 & 20.49 & $33.64 *$ & $53.13 * *$ & -12.34 & -16.99 & -10.33 & -7.02 & -3.29 & -4.59 & -2.13 & 0.67 \\
\hline 36 & Utkal Pragnya x PKM-1 & 12.46 & 5.79 & 13.48 & 30.03 & 6.65 & 4.75 & 1.15 & 4.88 & 3.01 & 1.53 & 4.34 & 7.32 \\
\hline 37 & Utkal Pragnya x Arka Meghali & 9.22 & 6.44 & 14.18 & 30.83 & 1.78 & 0.00 & -3.44 & 0.12 & -6.42 & -6.94 & -7.12 & -4.47 \\
\hline 38 & Utkal Pragnya x Anagha & 3.60 & -6.50 & 0.30 & 14.93 & -15.25 & $-20.94 *$ & -11.82 & -8.57 & 1.93 & 1.00 & 2.68 & 5.61 \\
\hline 39 & Arka Abha x Vaibhav & 2.96 & 2.11 & 13.25 & 29.77 & 1.50 & -11.68 & -4.59 & -1.07 & $-6.42 *$ & -6.76 & -4.34 & -1.62 \\
\hline 40 & Arka Abha x PKM-1 & $59.73 * *$ & $49.08 * *$ & $62.64 * *$ & $86.35 * *$ & 13.56 & 5.55 & -1.72 & 1.9 & $-8.58 * *$ & $-8.99 *$ & -6.47 & -3.80 \\
\hline 41 & Arka Abha x Arka Meghali & $62.50 * *$ & $57.08 * *$ & $71.36^{* *}$ & $96.35 * *$ & 6.89 & -0.68 & -7.46 & -4.05 & -1.94 & -3.45 & -1.66 & 1.14 \\
\hline 42 & Arka Abha x Anagha & $34.58^{*}$ & 20.56 & $31.52^{*}$ & $50.70 * *$ & 3.12 & -11.47 & -1.26 & 2.38 & -3.00 & -3.09 & -1.29 & 1.52 \\
\hline 43 & Arka Abha x Utkal Pragnya & 3.49 & 2.63 & 11.95 & 28.28 & 10.24 & 0.77 & -2.70 & 0.89 & 2.29 & 1.27 & 3.14 & 6.08 \\
\hline 44 & Arka Saurabh x Vaibhav & -13.35 & -15.70 & -1.14 & 13.28 & $-21.20 *$ & $-26.55^{*}$ & -20.65 & -17.73 & 1.77 & -1.62 & 0.92 & 3.80 \\
\hline 45 & Arka Saurabh x PKM-1 & -8.37 & -17.25 & -2.95 & 11.20 & 1.54 & 1.41 & -5.34 & -1.84 & -5.40 & $-8.63^{*}$ & -6.10 & -3.42 \\
\hline 46 & Arka Saurabh x Arka Meghali & -10.79 & -16.67 & -2.27 & 11.98 & 9.69 & 9.59 & 2.29 & 6.07 & 2.66 & 1.12 & -0.18 & 2.66 \\
\hline 47 & Arka Saurabh x Anagha & 3.57 & -10.08 & 5.45 & 20.83 & 6.80 & -1.90 & 9.41 & 13.44 & $-9.64 * *$ & $-12.27 * *$ & $-10.81 * *$ & $-8.27 *$ \\
\hline 48 & Arka Saurabh x Utkal Pragnya & -1.38 & -5.58 & 10.73 & 26.88 & 5.62 & 3.86 & 0.29 & 3.99 & -2.46 & -4.44 & -4.62 & -1.90 \\
\hline 49 & Arka Saurabh x Arka Abha & 17.27 & 13.18 & $32.73^{*}$ & $52.08 * *$ & 2.35 & -4.98 & -11.30 & -8.03 & -1.22 & -4.17 & -2.40 & 0.38 \\
\hline 50 & Utkal Raja x Vaibhav & 5.00 & 3.28 & 14.55 & 31.25 & 14.17 & 11.14 & 20.14 & $24.57^{*}$ & -3.37 & -4.50 & -2.03 & 0.76 \\
\hline 51 & Utkal Raja x PKM-1 & $68.96 * *$ & $58.94 * *$ & $70.50 * *$ & $95.36 * *$ & $57.62 * *$ & $50.56^{*}$ & $54.10 * *$ & $59.79 * *$ & -0.91 & -2.16 & 0.55 & 3.42 \\
\hline 52 & Utkal Raja x Arka Meghali & $49.85 * *$ & $46.04 * *$ & $56.66 * *$ & $79.51 * *$ & -12.79 & -16.70 & -14.74 & -11.60 & 0.74 & 0.00 & 0.18 & 3.04 \\
\hline 53 & Utkal Raja x Anagha & $49.67 * *$ & $35.08 *$ & $44.91 * *$ & $66.04 * *$ & -6.27 & -9.77 & 0.63 & 4.34 & -5.86 & -6.55 & -4.99 & -2.28 \\
\hline 54 & Utkal Raja x Utkal Pragnya & -1.69 & -1.69 & 5.45 & 20.83 & $-34.00 * *$ & $-27.46^{* *}$ & $-25.76^{*}$ & -23.02 & 3.88 & 3.69 & 3.88 & 6.84 \\
\hline 55 & Utkal Raja x Arka Abha & 13.87 & 12.92 & 23.18 & $41.15^{*}$ & -0.50 & -11.43 & -9.41 & -6.07 & $-6.50 *$ & -7.26 & -5.55 & -2.85 \\
\hline 56 & Utkal Raja x Arka Saurabh & 21.46 & 16.28 & $36.36^{*}$ & $56.25 * *$ & -8.79 & -12.78 & -11.07 & -7.79 & 0.75 & -1.48 & -1.29 & 1.52 \\
\hline & SEm \pm & 7.06 & 8.15 & \multicolumn{2}{|c|}{8.15} & & 0.82 & 0.94 & 0.94 & & 0.84 & 0.97 & 0.97 \\
\hline & CD@5\% & 14.49 & 16.73 & \multicolumn{2}{|c|}{16.73} & & 1.68 & 1.94 & 1.94 & & 1.73 & 2.00 & 2.00 \\
\hline & CD@1\% & 18.76 & 21.66 & \multicolumn{2}{|c|}{21.66} & & 2.18 & 2.52 & 2.52 & & 2.24 & 2.59 & 2.59 \\
\hline
\end{tabular}


Table.2 Estimation of mid parent, better parent and standard heterosis of $8 \times 8$ full diallel crosses in tomato for number of fruits per plant, average fruit weight and yield per plant

\begin{tabular}{|c|c|c|c|c|c|c|c|c|c|c|c|c|c|}
\hline \multirow{2}{*}{$\begin{array}{l}\text { SI. } \\
\text { No. }\end{array}$} & \multirow[t]{2}{*}{ Hybrids } & \multicolumn{4}{|c|}{ Number of fruits per plant } & \multicolumn{4}{|c|}{ Average fruit weight (g) } & \multicolumn{4}{|c|}{ Yield (kg/plant) } \\
\hline & & MP & BP & Check1 & Check2 & MP & $\mathrm{BP}$ & Check1 & Check2 & MP & $\mathrm{BP}$ & Check1 & Check2 \\
\hline 1 & Vaibhav x PKM-1 & 18.08 & -13.04 & 19.86 & 57.26 & -8.62 & $-24.18^{*}$ & 15.53 & 4.53 & $98.97 * *$ & $61.00 *$ & $57.49^{*}$ & $112.50 * *$ \\
\hline 2 & Vaibhav x Arka Meghali & 35.44 & 12.90 & 10.32 & 44.74 & $-39.70 * *$ & $-53.18 * *$ & -28.65 & $-5.44 * *$ & 8.55 & 5.86 & -35.97 & -13.60 \\
\hline 4 & Vaibhav x Utkal Pragnya & 32.01 & 12.49 & 4.11 & 36.60 & $-36.12^{* *}$ & $-53.09 * *$ & -28.51 & $-35.32 * *$ & -6.02 & -15.22 & -36.24 & -13.97 \\
\hline 5 & Vaibhav x Arka Abha & 8.74 & -2.61 & -19.77 & 5.26 & $-35.45^{* *}$ & $-44.01 * *$ & -14.68 & -22.80 & 35.20 & 15.24 & -1.09 & 33.46 \\
\hline 8 & PKM-1 x Arka Meghali & -28.83 & -39.19 & -16.19 & 9.96 & $-60.16^{* *}$ & $-63.37 * *$ & $-63.19 * *$ & $-66.70 * *$ & -51.23 & $-61.28 *$ & $-62.13 *$ & -48.90 \\
\hline 9 & PKM-1 x Anagha & $-80.67 *$ & 62.45 & $123.90^{*}$ & $193.76^{* *}$ & $-36.83^{*}$ & $-45.68 * *$ & $-45.42 * *$ & $-50.61 * *$ & $109.86^{* *}$ & $66.02 * *$ & $62.40^{*}$ & $119.12 * *$ \\
\hline 10 & PKM-1 x Utkal Pragnya & $81.77 *$ & 51.91 & $109.37^{*}$ & $174.70^{* *}$ & $-35.47^{*}$ & $-44.80 * *$ & $-44.54 * *$ & $-49.82 * *$ & 34.80 & 19.22 & 16.62 & 57.35 \\
\hline 11 & PKM-1 x Arka Abha & 23.81 & -1.09 & 36.32 & 78.85 & $-25.42 *$ & $-29.25^{*}$ & -20.77 & $-28.31 *$ & 41.54 & 32.87 & 29.97 & $75.37^{*}$ \\
\hline 12 & PKM-1 x Arka Saurabh & 3.84 & -4.37 & 31.81 & 72.93 & $-32.61 * *$ & $-35.08 *$ & $-29.61 *$ & $-36.32 * *$ & 6.96 & 0.56 & -1.63 & 32.72 \\
\hline 13 & PKM-1 x Utkal Raja & 10.65 & -34.51 & -9.74 & 18.42 & 8.57 & -18.21 & -17.82 & -25.64 & 37.78 & -22.28 & -23.98 & 2.57 \\
\hline 17 & Arka Meghali x Arka Saurabh & 0.00 & -7.90 & 6.88 & 40.23 & -17.89 & -27.02 & -20.88 & $-28.42 *$ & 21.82 & 1.58 & -12.53 & 18.01 \\
\hline 18 & Arka Meghali x Utkal Raja & $155.27 *$ & 60.70 & 57.02 & 106.02 & -6.22 & -24.79 & $-36.61^{*}$ & $-42.64 * *$ & $105.84^{* *}$ & $86.26^{*}$ & 7.08 & 44.49 \\
\hline 19 & Anagha x Utkal Pragnya & 72.80 & 59.08 & 75.03 & 129.64 & -33.84 & -34.26 & $-52.45 * *$ & $-56.98 * *$ & 20.82 & 6.16 & -20.16 & 7.72 \\
\hline 20 & Anagha x Arka Abha & 67.00 & 46.02 & 60.66 & 110.79 & $-35.22 *$ & $-46.69 * *$ & $-40.30 * *$ & $-45.99 * *$ & $63.74 *$ & 36.19 & 16.89 & 57.72 \\
\hline 21 & Anagha x Arka Saurabh & 17.62 & 14.57 & 32.95 & 74.44 & -24.98 & $-37.47 * *$ & $-32.21^{*}$ & $-38.66 * *$ & 29.52 & 7.59 & -7.36 & 25.00 \\
\hline 22 & Anagha x Utkal Raja & 35.49 & -16.67 & -8.31 & 20.30 & -2.47 & -16.92 & $-39.91 * *$ & $-45.63 * *$ & 30.98 & -20.10 & $-54.50^{*}$ & -38.60 \\
\hline 23 & Utkal Pragnya x Arka Abha & 23.54 & 16.75 & 8.05 & 41.77 & $-56.96^{* *}$ & $-64.76^{* *}$ & $-60.53 * *$ & $-64.29 * *$ & -49.24 & -52.38 & $-59.13^{*}$ & -44.85 \\
\hline 24 & Utkal Pragnya x Arka Saurabh & -7.28 & -16.67 & -3.30 & 26.88 & $-45.80^{* *}$ & $-55.05^{* *}$ & $-51.27 * *$ & $-55.91 * *$ & -46.62 & -50.00 & $-56.95^{*}$ & -41.91 \\
\hline 25 & Utkal Pragnya x Utkal Raja & 69.20 & 7.74 & -0.29 & 30.83 & -21.73 & -32.97 & $-52.13 * *$ & $-56.69 * *$ & -5.59 & -44.93 & $-58.58^{*}$ & -44.12 \\
\hline 26 & Arka Abha x Arka Saurabh & -26.56 & -37.21 & -27.13 & -4.40 & -15.13 & -16.48 & -6.47 & -15.37 & 29.64 & 29.43 & 11.44 & 50.37 \\
\hline 27 & Arka Abha x Utkal Raja & 80.92 & 18.26 & -2.58 & 27.82 & 7.32 & -21.95 & -12.59 & -20.91 & $111.63^{*}$ & 21.27 & 4.09 & 40.44 \\
\hline
\end{tabular}


Int.J.Curr.Microbiol.App.Sci (2018) 7(1): 1073-1080

\begin{tabular}{|c|c|c|c|c|c|c|c|c|c|c|c|c|c|}
\hline 28 & Arka Saurabh x Utkal Raja & -35.42 & -60.67 & -54.36 & -40.11 & 7.55 & -20.98 & -14.33 & -22.48 & 3.87 & -40.51 & -48.77 & -30.88 \\
\hline 29 & PKM-1 x Vaibhav & -26.14 & -45.60 & -25.03 & -1.64 & $-40.86 * *$ & $-50.93 * *$ & -25.23 & $-32.35^{*}$ & -25.65 & -39.83 & -41.14 & -20.59 \\
\hline 30 & Arka Meghali x Vaibhav & -6.19 & -21.80 & -23.60 & 0.24 & $-29.25 * *$ & $-45.06 * *$ & -16.28 & -24.25 & 32.10 & 28.83 & -22.07 & 5.15 \\
\hline 31 & Arka Meghali x PKM-1 & -23.18 & -34.36 & -9.53 & 18.70 & -11.20 & -18.35 & -17.96 & -25.77 & 7.02 & -15.04 & -16.89 & 12.13 \\
\hline 32 & Anagha x Vaibhav & $119.46^{*}$ & 74.74 & 92.26 & $152.26^{*}$ & -2.94 & $-28.44 * *$ & 9.05 & -1.33 & $112.53 * *$ & $106.60 * *$ & 24.80 & $68.38^{*}$ \\
\hline 33 & Anagha x PKM-1 & 32.17 & 18.85 & 63.80 & 114.91 & 15.23 & -0.92 & -0.44 & -9.91 & 44.01 & 13.93 & 11.44 & 50.37 \\
\hline 34 & Anagha x Arka Meghali & 47.35 & 39.10 & 53.05 & 100.81 & $-44.15 * *$ & $-48.11 * *$ & $-56.26 * *$ & $-60.43 * *$ & 20.00 & 19.43 & -31.34 & -7.35 \\
\hline 35 & Utkal Pragnya x Vaibhav & 23.40 & 5.15 & -2.68 & 27.69 & $-25.64 *$ & $-45.40 * *$ & -16.79 & -24.71 & 58.23 & 42.75 & 7.36 & 44.85 \\
\hline 36 & Utkal Pragnya x PKM-1 & 36.19 & 13.83 & 56.88 & 105.83 & $-36.08^{*}$ & $-45.32 * *$ & $-45.06^{* *}$ & $-50.29 * *$ & 20.94 & 6.96 & 4.63 & 41.18 \\
\hline 37 & Utkal Pragnya x Arka Meghali & 34.53 & 30.98 & 27.98 & 67.91 & -32.66 & $-37.80 *$ & $-47.57 * *$ & $-52.56 * *$ & 3.90 & -8.33 & -31.06 & -6.99 \\
\hline 38 & Utkal Pragnya x Anagha & -21.50 & -27.73 & -20.49 & 4.32 & $-38.44 *$ & -38.83 & $-55.76 * *$ & $-59.97 * *$ & -49.69 & -55.80 & $-66.76 * *$ & -55.15 \\
\hline 39 & Arka Abha x Vaibhav & 16.21 & 4.09 & -14.26 & 12.50 & $-39.27 * *$ & $-47.32 * *$ & -19.73 & $-27.37 *$ & 11.73 & -4.76 & -18.26 & 10.29 \\
\hline 40 & Arka Abha x PKM-1 & -2.77 & -22.33 & 7.05 & 40.45 & -11.13 & -15.70 & -5.59 & -14.58 & 28.49 & 20.61 & 17.98 & 59.19 \\
\hline 41 & Arka Abha x Arka Meghali & -23.85 & -29.82 & -31.43 & -10.04 & -14.86 & -25.39 & -16.44 & -24.40 & -13.31 & -27.62 & -37.87 & -16.18 \\
\hline 42 & Arka Abha x Anagha & 68.52 & 47.34 & 62.12 & 112.71 & $-40.89 * *$ & $-51.36 * *$ & $-45.53 * *$ & $-50.71 * *$ & 32.82 & 10.48 & -5.18 & 27.94 \\
\hline 43 & Arka Abha x Utkal Pragnya & 12.20 & 6.04 & -1.86 & 28.76 & $-37.35 * *$ & $-48.70 * *$ & $-42.55 * *$ & $-48.02 * *$ & -24.87 & -29.52 & 39.51 & -18.38 \\
\hline 44 & Arka Saurabh x Vaibhav & -47.83 & -59.26 & -52.72 & -37.97 & $-43.11 * *$ & $-51.32 * *$ & -25.82 & $-32.88^{*}$ & -53.16 & $-60.13^{*}$ & $-65.67 * *$ & -53.68 \\
\hline 45 & Arka Saurabh x PKM-1 & -32.28 & -37.63 & -14.04 & 12.78 & -19.15 & -22.11 & -15.56 & -23.60 & 20.00 & 12.81 & 10.35 & 48.90 \\
\hline 46 & Arka Saurabh x Arka Meghali & -55.98 & -59.46 & -52.95 & -38.27 & $-41.15^{* *}$ & $-47.70 * *$ & $-43.30 * *$ & $-48.70 * *$ & $-69.64 *$ & $-74.68 * *$ & $-78.20 * *$ & $-70.59 *$ \\
\hline 47 & Arka Saurabh x Anagha & 49.24 & 45.37 & 68.70 & 121.33 & $-38.62 * *$ & $-48.83 * *$ & $-44.53 * *$ & $-49.81 * *$ & $117.90 * *$ & $81.01 * *$ & $55.86^{*}$ & $110.29 * *$ \\
\hline 48 & Arka Saurabh x Utkal Pragnya & -20.33 & -28.40 & -16.91 & 9.02 & $-29.37 *$ & $-41.42 * *$ & $-36.50^{*}$ & $-42.54 * *$ & -15.20 & -20.57 & -31.6 & -7.72 \\
\hline 49 & Arka Saurabh x Arka Abha & -32.95 & -42.68 & -33.48 & -12.73 & $-34.68 * *$ & $-35.73 * *$ & -28.02 & $-34.87 * *$ & 7.45 & 7.28 & -7.63 & 24.63 \\
\hline 50 & Utkal Raja x Vaibhav & 15.56 & -19.78 & -47.71 & -31.39 & $-50.75^{* *}$ & $-67.15 * *$ & $-49.94 * *$ & $-54.71 * *$ & 28.36 & -22.52 & $-53.13^{*}$ & -36.76 \\
\hline 51 & Utkal Raja x PKM-1 & -9.25 & -46.29 & -25.97 & -2.88 & 16.64 & -12.14 & -11.71 & -20.12 & 26.91 & -28.41 & -29.97 & -5.51 \\
\hline 52 & Utkal Raja x Arka Meghali & -7.81 & -41.96 & -43.30 & -25.60 & 11.28 & -10.75 & -24.78 & $-31.94 *$ & 108.56 & 27.01 & -26.98 & -1.47 \\
\hline 53 & Utkal Raja x Anagha & 17.94 & -27.46 & -20.19 & 4.72 & -27.28 & -38.05 & $-55.19 * *$ & $-59.46 * *$ & 33.33 & -18.66 & $-53.68 *$ & -37.50 \\
\hline 54 & Utkal Raja x Utkal Pragnya & -11.27 & -43.50 & -47.71 & -31.39 & $-48.40^{*}$ & $-55.81 * *$ & $-68.44 * *$ & $-71.45^{* *}$ & -61.49 & $-77.54 *$ & $-83.11 * *$ & $-77.21 *$ \\
\hline 55 & Utkal Raja x Arka Abha & 77.20 & 15.83 & -4.58 & 25.19 & -18.67 & $-40.85 * *$ & $-33.76^{*}$ & $-40.07 * *$ & 19.11 & -31.75 & -41.42 & -20.96 \\
\hline 56 & Utkal Raja x Arka Saurabh & -17.91 & -50.00 & -41.98 & -23.87 & 11.47 & -18.09 & -11.20 & -19.66 & 28.18 & -26.58 & -36.78 & -14.71 \\
\hline & SEm \pm & 15.03 & 17.36 & \multicolumn{2}{|c|}{17.36} & 7.87 & 9.09 & \multicolumn{2}{|c|}{9.09} & 0.37 & 0.43 & \multicolumn{2}{|c|}{0.43} \\
\hline & CD@5\% & 30.85 & 35.62 & \multicolumn{2}{|c|}{35.62} & 16.16 & 18.66 & \multicolumn{2}{|c|}{18.66} & 0.77 & 0.89 & \multicolumn{2}{|c|}{0.89} \\
\hline & CD@1\% & 39.94 & 46.12 & \multicolumn{2}{|c|}{46.12} & 20.92 & 24.16 & \multicolumn{2}{|c|}{24.16} & 1.00 & 1.16 & \multicolumn{2}{|c|}{1.16} \\
\hline
\end{tabular}

1078 
The best combinations for economic characters

\begin{tabular}{|c|c|c|}
\hline Sl. No. & Character & Cross combination \\
\hline \multirow[t]{3}{*}{1} & \multirow[t]{3}{*}{ Yield per plant (More than $2500 \mathrm{~g}$ ) } & Vaibhav x PKM-1 \\
\hline & & PKM-1 x Anagha \\
\hline & & Arka Sourabh x Anagha \\
\hline \multirow[t]{3}{*}{2} & \multirow{3}{*}{$\begin{array}{l}\text { Number of fruits per plant (More than } 60 \text { fruits } \\
\text { per plant) }\end{array}$} & PKM-1 x Anagha \\
\hline & & PKM-1 x Utkal Pragnya \\
\hline & & Vaibhav x Anagha \\
\hline \multirow[t]{2}{*}{3} & \multirow[t]{2}{*}{ Average fruit weight (More than $70 \mathrm{~g}$ ) } & Vaibhav x Arka Saurabh \\
\hline & & Vaibhav x PKM-1 \\
\hline
\end{tabular}

Yield is a composite character, evidences suggest that heterosis of such a complex character is much regulated by the vigour expressed by its component character, such as average fruit weight and number of fruits per plant. In the present investigation, the yield per plant increased mainly due to increase in average fruit weight and number of fruits per plant.

Except fourteen crosses out of fifty six hybrids manifested positive heterosis over mid-parent. Twenty five crosses showed negative heterosis over better parent. The highest mean value for this trait is shown by the hybrid PKM-1 x Anagha (2.98) is higher than that of best parent PKM-1 (1.79). Only three hybrids exhibited significant positive heterosis over check- 1 and over check-2, five hybrids showed significantly positive heterosis. All these crosses except one have high yielding PKM-1 as one of their parent.

The foregoing discussion on yield and yield components indicated the role of non-additive and additive gene effect in governing these traits.

Heterosis never concerns directly to the whole plant organization, but occurs in the development of individual traits. Therefore, the sources of heterosis are essentially formed and located in separate systems, enclosed in the cell apparatus of the hybrid progenies.
These sources represent on altered genetic system brought about by the interaction of heterogeneous genetic background.

The interaction does so through complementation and mutual intensification of structural genes (such as changes in gene dose, position effect, production of extra DNA copies etc.) and through improvement of the balance of genetic factors controlling the regulatory mechanisms of the cell.

\section{References}

Kulkarni, G.P., 2003. Investigations on bacterial wilt resistance in tomato. Ph.D. Thesis. Uni. Agric. Sci., Dharwad (India).

Mahendrakar, P., 2004. Development of $\mathrm{F}_{1}$ hybrids in tomato (Lycopersicum esculentum Mill.). M.Sc. (Agri.) Thesis, Univ. Agric. Sci., Dharwad.

Duhan, D., Partap, P.S., Rana, M.K. and Basawana, K.S., 2005. Study of heterosis for growth and yield characters in tomato. Haryana J. Hort. Sci., 34: 366-370.

Thakur, A. K., Kholi, U. K., and Joshi, A., 2004. Evaluation of diallel progeny and heterois for yield and yield components in tomato (Lycopersicon esculentum Mill.) Haryana J. Hort. Sci. 33(1\&2): 106-108.

Tiwari, A. and Lal, G., 2004. Studies on 
heterosis for quantitative and qualitative characters in tomato (Lycopersicon esculentum Mill.). Progr. Horti., 36(1): $122-127$.

\section{How to cite this article:}

Jyothi Kattegoudar, H.B. Lingaiah, N.C. Mamtha, Ambresh and Arunkumar, B. 2018. Heterosis Studies for Yield and Yield Attributing Characters of Tomato (Solanum lycopersicum L.). Int.J.Curr.Microbiol.App.Sci. 7(01): 1073-1080. doi: https://doi.org/10.20546/ijcmas.2018.701.130 